\title{
Minimally invasive therapy of perforations at the esophagogastric junction by over-the-scope clipping
}

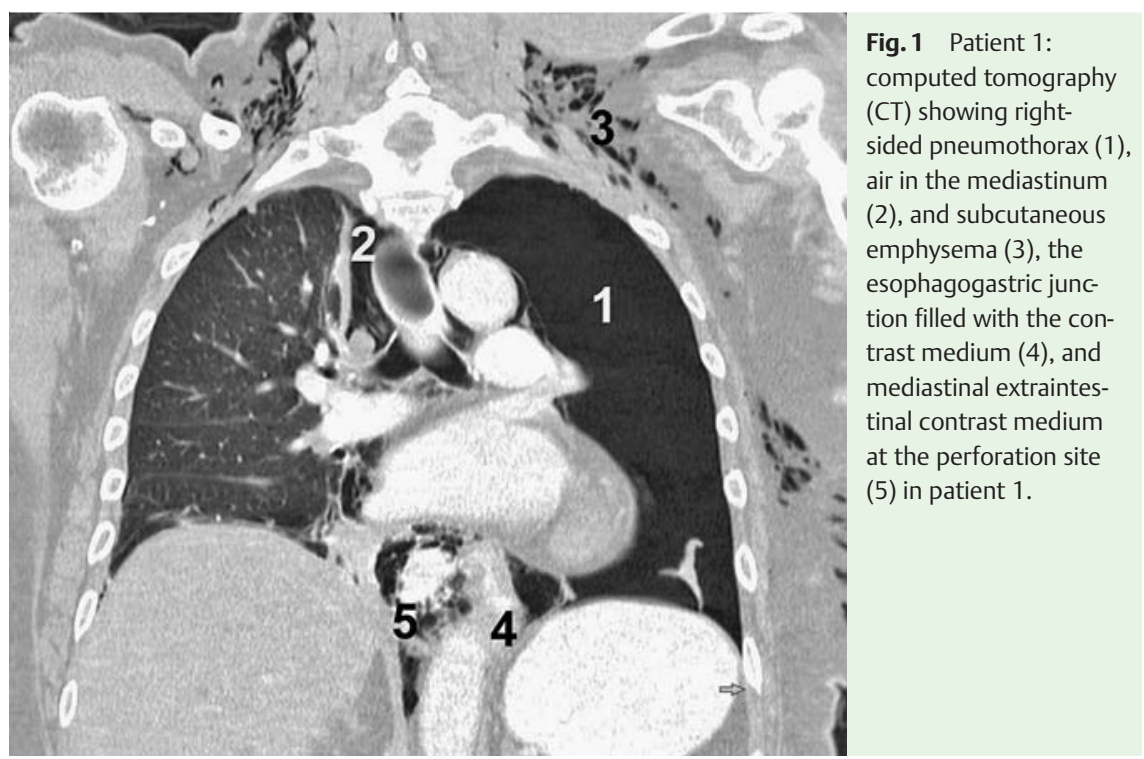

Perforations of the esophagogastric junction remain a problem for endoscopic therapy, as self-expandable stents often tend to migrate because of the discrepancy between the stent diameter and the larger diameter of the proximal stomach, thus failing to seal leaks at the esophagogastric junction [1-3]. Treatment using over-the-scope clips (OTSC) (Ovesco AG, Tübingen, Germany) has been described for perforations at different gastrointestinal locations but not at the esophagogastric junction $[4,5]$. Here we report on the use of OTSC in three patients with perforations at the esophagogastric junction. In two patients, the perforations were iatrogenic (occurring after endoscopic retrograde cholangiopancreatography [ERCP]), and the third patient had Boerhaave syndrome. The perforations were detected within 24 hours of development in all three patients. The diagnosis was confirmed with computed tomography (CT), which revealed pneumomediastinum and subcutaneous emphysema ( Fig. 1). In two patients, CT showed evidence of pneumothorax with basal pleural effusion and thoracic drains were placed before endoscopy. On endoscopy the perforations were noted to be at the esophagogastric junction. In patient $1, \mathrm{a}$ 10-mm perforation was seen immediately distal to the z-line ( $\mathbf{F i g . 2 a}$ ). In patient 2 , a $10-\mathrm{mm}$ perforation was verified crossing the $z$-line. In patient 3 , a perforating tear extended from the z-line downwards $4 \mathrm{~cm}$ into the stomach ( $\mathrm{Fig} .3 \mathrm{a}$ ). One OTSC $(11 / 6 \mathrm{t})$ was applied in patients 1 and 2, and two OTSC (11/6t) and four hemoclips (HX610090L, Olympus Optical Ltd., Tokyo, Japan) were used in patient 3 ( Fig.2b and Fig.3b). Postinterventional CT scan with esophagogastric contrast filling subsequently verified sufficient closure in all three patients. Because of persisting clinical sepsis caused by pleural empyema in patient 2 , right-sided video-assisted thoracic surgery (VATS) and pleural lavage and decortication was done 3 days after OTSC. The rest of the clinical course was uneventful in all three patients. They were dismissed on day 9 , 19 , and 8 after OTSC, respectively. None of them reported dysphagia or other symptoms which may have been attributed to the OTSC. All three patients underwent follow-up endoscopy 6-12 weeks after OTSC procedure ( $\bullet$ Fig. 2 c).

Endoscopy_UCTN_Code_TTT_1AO_2AI

Competing interests: None
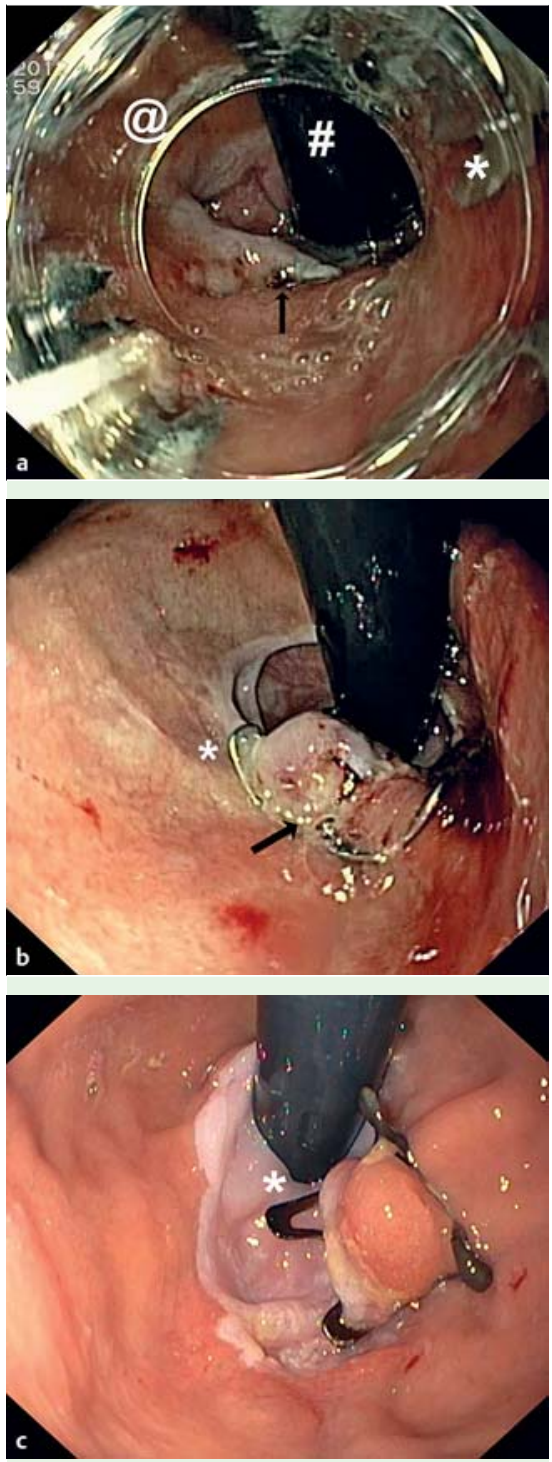

Fig. 2 Patient 1: retroflex endoscopic views. a An approximately 10 -mm long perforation (arrow) is seen immediately distal to the z-line at the cardia. Over-the-scope clip (OTSC) graspers $\left({ }^{*}\right)$ are visible at the 1 o'clock and 7 o'clock positions on the applicator device (@). (\#): Scope. b Closure of the same lesion (arrow) using one OTSC $\left(^{*}\right)$. c The lesion 6 weeks after OTSC: the clip $\left({ }^{*}\right)$ is still in place. 


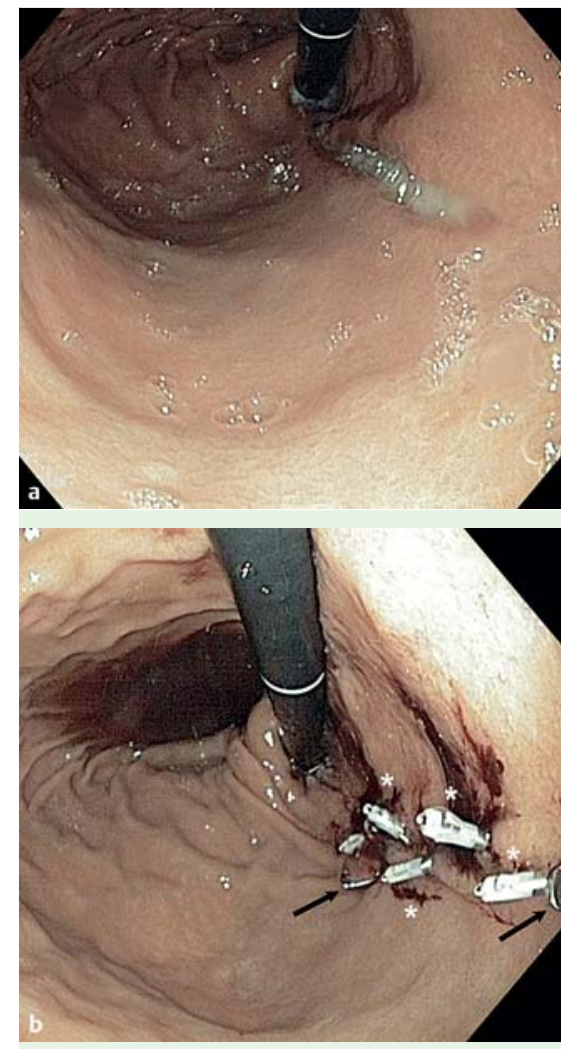

Fig. 3 Patient 3: retroflex endoscopic view. a An approximately 4-cm long perforation extending from the cardia into the lesser curvature of the proximal stomach. $\mathbf{b}$ Closure of the lesion in a using two OTSC (arrows) and four hemoclips $\left({ }^{*}\right)$.

\section{A. Braun, H. J. Richter-Schrag, \\ A. Fischer, J. Hoeppner}

Surgical Endoscopy, Department of Surgery, University of Freiburg, Germany

\section{References}

1 Shim CS. Esophageal stent for cervical esophagus and esophagogastric junction. Clin Endosc 2012; 45: 235-239

2 Fischer A, Thomusch 0 , Benz $S$ et al. Nonoperative treatment of 15 benign esophageal perforations with self-expandable covered metal stents. Ann Thorac Surg 2006; 81: 467-472

3 Fischer A, Bausch D, Richter-Schrag HJ. Use of a specially designed partially covered selfexpandable metal stent (PSEMS) with a 40 $\mathrm{mm}$ diameter for the treatment of upper gastrointestinal suture or staple line leaks in 11 cases. Surg Endosc 2012, [Epub ahead of print]

4 Kirschniak A, Subotova N, Zieker D et al. The over-the-scope clip (OTSC) for the treatment of gastrointestinal bleeding, perforations, and fistulas. Surg Endosc 2011; 25: 2901 2905

5 Sandmann M, Heike M, Faehndrich M. Application of the OTSC system for the closure of fistulas, anastomosal leakages and perforations within the gastrointestinal tract. Z Gastroenterol 2011; 49: 981 -985

\section{Bibliography}

DOI http://dx.doi.org/

10.1055/s-0032-1326449

Endoscopy 2013; 45: E133-E134

(c) Georg Thieme Verlag KG

Stuttgart · New York

ISSN 0013-726X

\section{Corresponding author}

\section{J. Hoeppner}

Department of Surgery

University of Freiburg

Hugstettter Str. 55

79106 Freiburg

\section{Germany}

Fax: +49-761-270-2804

jens.hoeppner@uniklinik-freiburg.de 